\title{
Treating Vestibular Schwannomas with Gamma-Knife Radiosurgery Versus Microsurgery: Long-Term Follow-up of 702 Cases
}

\author{
AHMED R. RIZK, M.D.*****; GUIHERME LEPSLI, M.D.*; GERHARD A. HORSTMANN, M.D.** and \\ MARCOS TATABIBA, M.D.* \\ The Department of Neurosurgery, Eberhard Karls University, Tübingen, Germany*, Gamma Knife Centre Krefeld, Krefeld, \\ Germany** and Department of Neurosurgery, Banha Faculty of Medicine, Benha University, Egypt***
}

\begin{abstract}
Background: The three treatment options currently considered for vestibular schwannoma are expectant treatment, microsurgery and radiosurgey, and to date no class I evidence favors one treatment over the others.

Aim of Study: The objective of this study was to compare long-term functional outcome after gamma knife radiosurgery versus surgical treatment.

Methods: A group of 275 patients submitted to microsurgery and 427 patients treated with radiosurgery in two different centers were followed-up for $15.2 \pm 13.6$ months and $45.6 \pm 26.8$ months, respectively. We assessed tumor volumetry based on serial MR images, facial nerve function, hearing function, tinnitus, trigeminal symptoms and vertigo, and compared them between treatment groups.

Results: Based on normalized volumetry over time, surgery was more efficient for tumor control than radiosurgery $(p<0.001)$. After radiosurgery, hearing and facial nerve functions remained stable regardless of tumor size. On the other hand, after surgery, there was clinical deterioration for both small and large tumors, yet the deterioration for small tumors was very discrete, rendering quite similar functional results across groups in this category. Furthermore, surgery alleviated symptoms like facial hypoesthesia, dizziness, and tinnitus more effectively than radiosurgery.
\end{abstract}

Conclusion: These results represent an important contribution to the field and offer evidence-based criteria to support the indication of a particular treatment modality according to the patient's expectations and professional activity.

Key Words: Vestibular schwannoma-Radiosurgery - Microsurgery - Gamma knife.

\section{Introduction}

THREE therapeutic options are currently considered for vestibular schwannoma (VS): Expectant treatment, microsurgery, and radiosurgery. No class I evidence exists to support one treatment over the others, and some clinical aspects are usually taken

Correspondence to: Dr. Ahmed R. Rizk, E-Mail: arizkrizk@gmail.com into consideration in the decision-making process. Expectant treatment may be chosen for small tumors, especially in elderly patients and in cases where the tumor shows no significant growth within the first year (i.e., $<2 \mathrm{~mm} /$ year), as monitored with MRI [1]. While surgery is offered for most cases, its benefits seem to be greatest for large tumors, which carry a greater risk of compression to the brainstem or displacement of cranial nerves. However, postsurgical hearing and facial nerve function are best preserved when the tumor is small or medium-sized [2] . Radiosurgery is considered a preferable treatment option in elderly patients, in cases with severe co-morbidities, and when the patient prefers not to have surgery, while it is usually not favored in cases of cystic or large tumors [3]. Large VSs represent a challenge to both surgical and radiosurgical treatment options, and the choice between treatments for small tumors remains controversial.

In fact, tumor size is a crucial factor when choosing the best treatment option, as it is also a major predictor of treatment results. Very few comparative studies published so far have addressed the clinical aspects supporting any one treatment modality. The aim of the present study was to compare the long-term functional results of two patient cohorts who had undergone either microsurgery or radiosurgery. Special attention was given to resection radicality (which was based on volumetric MRI), tumor recurrence or re-growth, facial nerve function and hearing function preservation, as well as recovery of additional symptoms such as tinnitus and facial hypoesthesia.

\section{Patients and Methods}

We performed a retrospective cohort study comparing two groups of patients harboring VSs. 
Patients in the first group were submitted to microsurgical resection by the senior author (MT) between 2004 and 2010 at the Department of Neurosurgery at the University of Tübingen, Germany, and patients in the second group were treated with gamma knife radiosurgery between 1997 and 2010 at the Gamma Knife Center in Krefeld, Germany. Both institutions have a busy VS program with more than 100 cases per year.

We enrolled 427 patients in the radiosurgery group (60.7\% women and $39.3 \%$ men), and 275 patients in the surgery group $(54.0 \%$ women and $46.0 \%$ men). Table 1 summarizes the clinical data, tumor size stratified according to the Hannover classification [4], as well as neurological status prior to treatment in both groups. Patients were followed-up for $45.6 \pm 26.7$ months after radiosurgery and for $15.3 \pm 14.4$ months after surgery (details regarding follow-up are provided in Table (2).

Surgical treatment followed a well-established technique [5], and was performed under general intravenous anesthesia without muscle relaxants. Anesthesia was induced with 0.4-1.0 $\mathrm{g} / \mathrm{kg}$ body weight sufentanil, and propofol $1 \%(2-4 \mathrm{mg} / \mathrm{kg}$ body weight). For maintenance, propofol was continued with $5 \mathrm{mg} / \mathrm{kg}$ body weight/hour and remifentanil with up to $0.5 \mathrm{~s} / \mathrm{k}$ body weight $/ \mathrm{min}$. As already described, $89 \%$ of patients were operated on in the semi-sitting position; resection was conducted using the microsurgical technique, through a retrosigmoid approach and under continuous electrophysiological monitoring (unilateral auditory evoked potentials, unilateral facial motor evoked potentials "MEP", contra-lateral median $\mathrm{n}$. and tibial $\mathrm{n}$. sensory evoked potentials, and MEPs of the contralateral thumb adductor and $\mathrm{m}$. tibial ant.). Patients were extubated immediately postoperatively, and on the following day, a CT scan was performed prior to discharge from the ICU.

Radiosurgery was performed in a Gamma Knife 4C unit (Elekta, Stockholm, Sweden). The patients were admitted on the day of the procedure, the Leksell stereotactic frame with insulated posts and titanium screws (Elekta, Stockholm, Sweden) was applied to the head under local anesthesia, and an axial 3D SPGR with i.v. Gadolinium $(1.0-1.5 \mathrm{~mm}$ thick) as well as a T2-weighed 3D image at 1.0$1.5 \mathrm{~mm}$ thickness in the ROI were obtained. Planning was performed using the Leksell Gamma Plan software (Elekta, Stockholm, Sweden). The prescribed dose at the margin was $13.0 \pm 0.2 \mathrm{~Gy}$, the prescription isodose was $63.7 \pm 4.0 \%$, the maximal dose was $20.5 \pm 1.5 \mathrm{~Gy}$, the mean coverage was $97 \%$, mean selectivity was $85 \%$, and the mean conformity index was 1.14 , for a mean target volume of 1.81 $\pm 2.21 \mathrm{~cm}^{3}$; the number of used shots was $18 \pm 10$, and the average treatment time was $37 \pm 16$ minutes.

The inclusion criterion was suspected diagnosis of VS, which was based on both clinical and radiological (MRI) findings. Patients were excluded if they had received prior treatment (either radiosurgery, microsurgery, or both), if they had suspected or confirmed neurofibromatosis, and/or if they were followed-up for less than six months after surgery and less than one year after radiosurgery.

We assessed the following variables: (i) Patient complaints; (ii) Facial nerve function based on the House-Brackmann (HB) grading scale; (iii) Hearing evaluation using the pure-tone audiogram; average pure tone and percentage of speech discrimination (all described in terms of the Gardner-Robertson "GR" grading scale); (iv) New symptoms or treatment-related complications (e.g., dizziness, tinnitus, facial hypoesthesia); and (v) Tumor volumetry based on thin-slice MRI scans $(0.7 \mathrm{~mm}$ thickness $\mathrm{T} 1$ with gadolinium). The volumetric study was performed on the Osirix 5.8 platform (Kanteron systems, NY, USA). Neurological assessment was repeated every 6 months, while hearing assessment and MRI scans were repeated once a year (these assessments were available for all patients at the time of the last follow-up). Data collection was performed by the second author (AR), who was not involved in data analysis.

This retrospective study was performed in line with the ethical rules of the involved centers (University of Tübingen and Krefeld Gamma Knife Center), with German federal laws, and with the most recent review of the Declaration of Helsinki.

\section{Statistical analyses:}

All statistical analyses were conducted using the SPSS 21.0.0 software (IBM Corp., NY, USA). Continuous, semi-continuous and semi-categorical data were initially compared to the normal curve by a K S distance test and a Shapiro test and classified as normal by their adherence to the Gaussian curve.

Parametric data are expressed as means and standard deviations. For independent samples, we used unpaired Student's $t$-tests, with or without a Welch correction, depending on Levene's test results. For dependent samples (over time) we used paired $t$-tests. 
Categorical data are expressed by absolute (n) and relative frequency (\%) and for the analysis of contingency matrices, we used Pearson's chi-square test. Additionally, we calculated the relative risks for clinical deterioration with their respective confidence intervals. A relative risk of treatment A over B above 1.0 indicates that the risk of deterioration in $\mathrm{A}$ is higher than in $\mathrm{B}$; a relative risk below 1.0, by contrast, indicates that the risk in $\mathrm{A}$ is lower than in B (i.e., a protective effect).

To assess tumor control, volumetric measurements were normalized to the pre-treatment value, and plotted for all patients as a function of time. A linear regression model was applied, using a least-squares algorithm.

For the entire study, alpha was set at $5 \%$.

\section{Results}

Groups did not differ from each other in terms of gender $(p=0.08)$, preoperative facial nerve function $(p=0.112)$ or incidence of tinnitus $(p=0.076)$. Tumor size, however, was significantly larger in the surgery group, which reflects the criteria used for treatment indication $(p<0.001)$. Preoperative hearing $(p<0.01)$, vestibular nerve $(p<0.01)$ and trigeminal nerve functions $(p<0.001)$ were also significantly more compromised in the surgery group (as a direct consequence of tumor size).

\section{Effectiveness of each treatment:}

First, we compared clinical outcome to pretreatment status in order to assess the effectiveness of each treatment modality in controlling tumorrelated symptoms. In general, radiosurgery was ineffective at relieving tinnitus, facial nerve function, and facial hypoesthesia $(p=0.134, p=0.731$, and $p=0.875$, respectively, Wilcoxon signed rank test after vs. pre-treatment). Radiosurgery also consistently resulted in reduced hearing $(p<0.001)$, but was efficient at improving dizziness $(p<0.001)$. By contrast, surgery significantly altered all functions evaluated: While facial nerve and hearing functions were significantly negatively affected following surgery, tinnitus, dizziness, and facial hypoesthesia significantly and consistently improved ( $p<0.001$ for all criteria).

\section{Tumor control:}

Next, we addressed the efficacy of both treatment modalities in controlling tumor growth. To this end, we normalized the volume measured at follow-up to the initial pre-treatment volume. As expected, surgery had a more drastic impact on tumor size (mean relative final volume for surgery:
$4 \pm 36 \%$, and radiosurgery: $125 \pm 175 \% ; p<0.001$, 2-tailed Mann-Whitney U). Nonetheless, $75 \%$ of patients treated with radiosurgery had a final relative volume smaller than $166 \%$ (an increase of only two-thirds of the initial volume over an observation period of 45.6 months), indicating that radiosurgery was effective in maintaining tumors relatively stable. Fig. (1) illustrates the relative volumetry (normalized to the preoperative size) in all patients submitted to radiosurgery (Fig. 1A) and surgery (1B). The histograms on the top part of graphs $1 \mathrm{~A}$ and $1 \mathrm{~B}$ represent the number of patients in each follow-up time interval. In the radiosurgery group, dots in the graph represent 1736 volumetric studies in 427 patients within a 150-month observation period. In 151 patients in the radiosurgery group, tumor size increased within the observation period (35.4\%). Transient swelling was observed in 88 patients, which began 3-18 months after radiation (mean of 6.7 months), followed by tumor shrinkage $8-85$ months after radiation (mean of 25.1 months). However, there was an overall tendency toward tumor reduction $(p<0.001)$.

In the surgery group $(n=275)$, the resulting curve was horizontal, indicating that surgery was very effective in controlling tumor growth. Specifically, total resection was not possible in only six cases, and there was no recurrence among those resected radically ( $99.6 \%$ control rate). Also, tumor residuals accounted for less than $5 \%$ of the initial volume. No cases required reoperation, but in 1 of these 6 cases, the tumor re-grew.

\section{Hearing function:}

At the last follow-up, patients in the radiosurgery group performed better than operated patients in terms of hearing function ( $p<0.001$, Pearson's Chi-square). As assessed by the Gardner-Robertson (GR) scale, $33.8 \%$ of patients had good or serviceable hearing after radiosurgery (i.e., GR grades I and II; $47.1 \%$ pre-treatment). On the other hand, only $25.0 \%$ of patients in the surgery group had a similar functional level after treatment (42.4\% presurgery; see Table 1). Considering only those patients with serviceable hearing prior to treatment (GR I and II), 59.1\% still had serviceable hearing after surgery, as compared with $72.0 \%$ of patients treated with radiosurgery (see also Fig. 3). Therefore, serviceable hearing was better preserved after radiosurgery than after surgery $(p<0.001)$. Considering that tumors in the surgical series were larger on average, we analyzed hearing preservation according to tumor size category (Fig. 4). This analysis revealed that, hearing preservation rates were comparable between surgery and radiosurgery 
for small tumors (see "small versus large tumors", below).

Finally, surgery was associated with an overall positive relative risk of developing non-serviceable hearing (mean 1.30, CI 95\% 1.15-1.47). Surprisingly, in the irradiated population, there was a similar positive risk for non-serviceable hearing (mean 1.25 , CI95\% 1.11-1.39).

\section{Facial nerve function:}

At the last follow-up, patients in the radiosurgery group had relatively better facial nerve function than operated patients $(p<0.001$, Pearson's chi-square), as assessed by the House-Brackmann (HB) scale. In the radiosurgery group, $97.2 \%$ of patients were classified as HB I and II (97.5\% pretreatment), whereas in the surgery group, $93.0 \%$ were classified as HB I or II (97.1\% pre-treatment). Therefore, although facial paralysis did occur in some patients post-operatively, it was usually not severe.

Repeating the same analysis performed for hearing, we observed that facial nerve function remained stable after radiosurgery $(0.04 \pm 0.40)$ but showed a trend toward deterioration after surgery $(-0.33 \pm 0.78, p<0.001$, Mann-Whitney U). When we analyzed the results in terms of tumor size, we observed that the overall deterioration after surgery was influenced by the larger tumors and that there was little difference between radiosurgery and surgery for the smaller tumors (see text, "small versus large tumors", and also Fig. 5).

There was a positive relative risk for severe facial nerve palsy associated with surgery (mean 2.75 , CI95\% 1.25-6.07). In radiosurgery, by contrast, the interval included the value 1 (mean 1.10, CI95\% 0.47-2.57), which indicates that the risk of severe facial nerve palsy was not significant.

\section{Other symptoms:}

Next, we evaluated the relative risk of developing new symptoms after either type of treatment, which revealed a protective effect (relative risk below 1) of surgery relative to radiosurgery with respect to facial hypoesthesia, dizziness and tinnitus (all $p s<0.001$, Pearson's chi-square; see Fig. 2, Table 1).

\section{Small versus large tumors:}

Since tumor size influences the choice of treatment, tumors in patients in the surgery group were consistently larger. Therefore, we further analyzed patients by stratifying them into small and large tumor groups. We then analyzed the ROC curves, setting the final relative volume as the outcome variable. The cut-off value for size was $2.5(\leq 2$ and $\geq 3$ ). Following the Hannover classification for vestibular schwannomas, small tumors in our group were classified as grades I and II, whereas large tumors were classified as grades IIIa, IIIb, IVa and $\mathrm{IVb}$ (see Fig. 2).

The two small tumor groups (radiosurgery and surgery) did not differ significantly from each other in terms of gender ( $p=0.082$, Chi square), size stratification according to the Hannover classification ( $p=0.305$, Mann Whitney $\mathrm{U})$, facial nerve function ( $p=0.195$, Mann Whitney $\mathrm{U}$ ) and hearing function ( $p=0.925$, Mann Whitney $\mathrm{U}$ ), incidence of tinnitus ( $p=0.065$, Chi square), or trigeminal symptoms ( $p=0.266$, Chi square); however, the groups differed in terms of age $(p<0.001$, Mann Whitney $\mathrm{U})$ and incidence of dizziness $(p<0.05$, Chi square).

The two large tumor groups (radiosurgery and surgery), on the other hand, did not differ significantly from each other regarding gender ( $p=0.576$, Chi square), facial nerve ( $p=0.066$, Mann Whitney $\mathrm{U})$, hearing function $(p=0.355$, Mann Whitney $\mathrm{U})$, incidence of tinnitus ( $p=0.544$, Chi square), dizziness $(p=0.138$, Chi square), or trigeminal symptoms $(p=0.372$, Chi square), but differed in terms of age $(p<0.001$, Mann Whitney $\mathrm{U})$ and tumor size $(p<0.01$, Mann Whitney U, i.e., larger in the surgery group).

Our data show that radiosurgery was better than surgery in preserving facial nerve and hearing functions in both tumor groups (facial nerve for small and large tumors, $p<0.01$; hearing for small and large tumors, <0.001; Mann Whitney U). Indeed, considering pre- and post-treatment functional grades, hearing as well as facial nerve function remained stable after radiosurgery for both small and large tumors. On the other hand, surgery resulted in functional (hearing and facial nerve) deterioration, which was more intense in the larger tumor population. Nevertheless, this deterioration (means, hearing: -0.9 and -1.1 ; facial nerve: -0.1 and -0.4 , for small and large tumors, respectively) was clinically not relevant in most cases. Indeed, when we analyzed hearing outcome according to tumor size (Fig. 4), we observed that $87 \%$ of patients with $\mathrm{T} 1$ tumors retained serviceable hearing after surgery, as compared with $74 \%$ of the irradiated patients. For T2, patients with serviceable hearing accounted for $62 \%$ of patients who underwent surgery and $56 \%$ who had radiosurgery. Results were worse for larger tumors in both groups, yet more so for the surgery group. However, the 
risk analysis revealed a similar risk of hearing loss (GR >3) between treatment groups when we considered the total series or the large tumor population, yet a higher risk of new hearing loss in the surgery group in the case of small tumors (see Fig. 2). A similar pattern was observed for the facial nerve, where $100 \%$ of $\mathrm{T} 1$ tumors and $97 \%$ of $\mathrm{T} 2$ tumors in the surgery group evolved to HB I, as compared with $96.5 \%$ of $\mathrm{T} 1$ tumors and $98.5 \%$ of T2 tumors in the radiation group (Fig. 5). In this case, the risk analysis indicated a higher risk for new apparent facial palsy in the large tumor group as well as in the entire surgery group (see Fig. 2). Taken together, these data indicate that both treat-

Table (1): Clinical characterization of VS patients prior to and after treatment.

\begin{tabular}{|c|c|c|c|c|c|}
\hline & \multicolumn{2}{|c|}{ Initial Presentation } & \multicolumn{2}{|c|}{ Final Presentation } & \multirow[b]{2}{*}{$\begin{array}{c}p- \\
\text { values }\end{array}$} \\
\hline & $\begin{array}{l}\text { Radio- } \\
\text { surgery }\end{array}$ & Surgery & $\begin{array}{l}\text { Radio- } \\
\text { surgery }\end{array}$ & Surgery & \\
\hline \multicolumn{6}{|l|}{ Gender, } \\
\hline \multicolumn{6}{|l|}{ No. $(\%)$ : } \\
\hline Female & $259(60.7)$ & $149(54)$ & & & \\
\hline Male & $108(39.3)$ & $126(46)$ & & & \\
\hline \multicolumn{6}{|l|}{ Size, No. (\%): } \\
\hline $\mathrm{T} 1$ & $58(13.6)$ & $22(8.0)$ & & & \\
\hline $\mathrm{T} 2$ & $137(32.1)$ & $70(25.4)$ & & & \\
\hline T3a & $99(23.2)$ & $53(19.6)$ & & & \\
\hline $\mathrm{T} 3 \mathrm{~b}$ & $65(15.2)$ & $51(18.5)$ & & & \\
\hline $\mathrm{T} 4 \mathrm{a}$ & $57(13.3)$ & $69(25.0)$ & & & \\
\hline $\mathrm{T} 4 \mathrm{~b}$ & $11(2.6)$ & $10(3.6)$ & & & \\
\hline $\begin{array}{l}\text { Facial nerve } \\
(H B), \%:\end{array}$ & & & & & $<0.001$ \\
\hline I & 97.0 & 94.2 & 96.0 & 74.3 & \\
\hline II & 0.5 & 2.9 & 1.2 & 17.8 & \\
\hline III & 0.7 & 1.4 & 0.7 & 5.4 & \\
\hline IV & 0.5 & 0.4 & 0.9 & 2.2 & \\
\hline $\mathrm{V}$ & 0.7 & 1.1 & 0.7 & 0.4 & \\
\hline VI & 0.5 & 0.0 & 0.2 & 0.0 & \\
\hline \multicolumn{6}{|l|}{$\begin{array}{l}\text { function }(G R) \text {, } \\
\% \text { : }\end{array}$} \\
\hline I & 16.2 & 8.0 & 12.9 & 2.2 & \\
\hline II & 30.9 & 34.4 & 20.9 & 22.8 & \\
\hline III & 38.2 & 45.7 & 43.2 & 21.4 & \\
\hline IV & 9.6 & 5.8 & 12.7 & 8.3 & \\
\hline $\mathrm{V}$ & 5.2 & 6.2 & 10.1 & 45.3 & \\
\hline Tinnitus, \%: & & & & & $<0.001$ \\
\hline Absent & 28.1 & 22.1 & 31.0 & 85.9 & \\
\hline Present & 71.9 & 77.9 & 69.0 & 14.1 & \\
\hline Dizziness, \%: & & & & & $<0.001$ \\
\hline Absent & 44.3 & 31.9 & 55.9 & 73.2 & \\
\hline Present & 55.7 & 68.1 & 44.1 & 26.8 & \\
\hline $\begin{array}{l}\text { Facial } \\
\text { hyposthesia, } \\
\% \text { : }\end{array}$ & & & & & $<.001$ \\
\hline Absent & 89.5 & 85.5 & 91.8 & 98.2 & \\
\hline Present & 10.5 & 14.5 & 8.2 & 1.8 & \\
\hline
\end{tabular}

ment modalities lead to similar hearing and facial nerve results in the case of small tumors.

In terms of tinnitus, surgery yielded better results than radiosurgery in both the small and large tumor groups (all $p s<0.001$ ). We also observed a relative advantage of surgery over radiosurgery for dizziness and facial hypoesthesia in the case of small tumors only $(p<0.001$ for both analyses, Chi-square test). For facial hypoesthesia, the difference between treatments practically disappeared with regard to large tumors. Finally, mean risk values above 1 indicate that surgery imposes an overall positive risk of facial palsy, especially in the large tumor population.

Table (2): Follow-up range and number of patients in both groups.

\begin{tabular}{lcc}
\hline Follow-up period & Radiosurgery N (\%) & Surgery N (\%) \\
\hline Less than 1 year & 0 & $138(50 \%)$ \\
1-2 years & $101(23.5 \%)$ & $80(29 \%)$ \\
2-3 years & $100(23.5 \%)$ & $35(13 \%)$ \\
3-5 years & $119(28 \%)$ & $15(6 \%)$ \\
5-7 years & $66(15.5 \%)$ & $7(3 \%)$ \\
7-10 years & $36(8.5 \%)$ & 0 \\
More than 10 years & $5(1 \%)$ & 0 \\
\hline Total & 427 & 275 \\
\hline
\end{tabular}
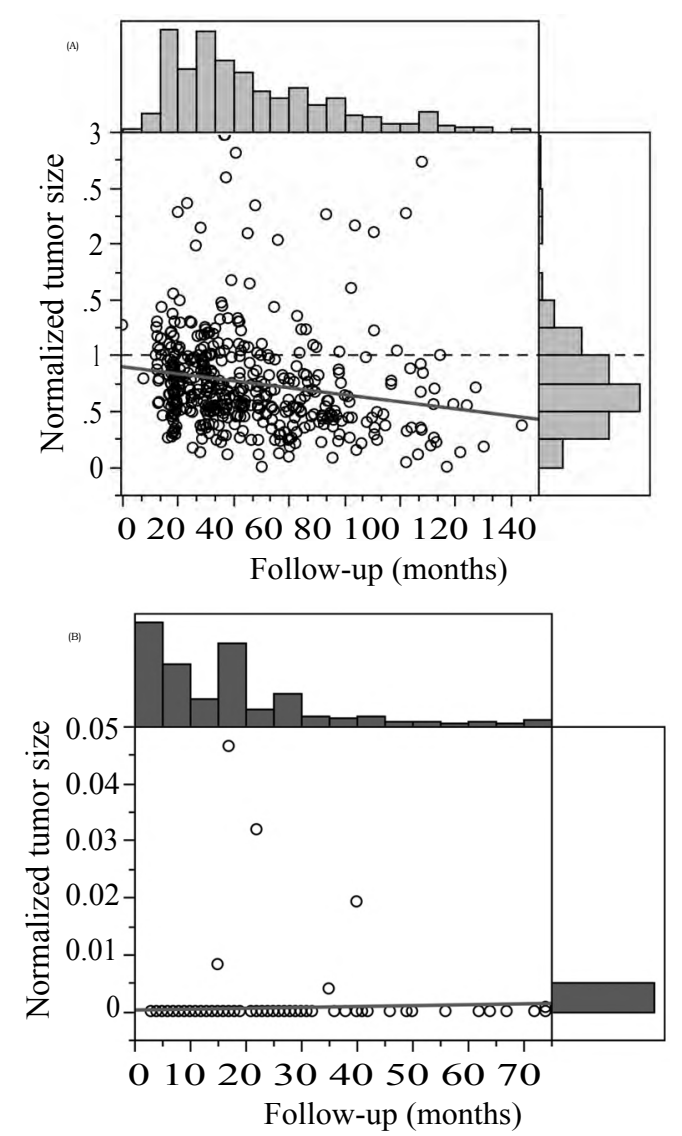

Fig. (1): Normalized tumor size (tumor size at follow-up relative to pre-treatment size). In A, cohort of patients submitted to radiosurgery. In $\mathrm{B}$, cohort of patients submitted to surgery. 
Relative risks

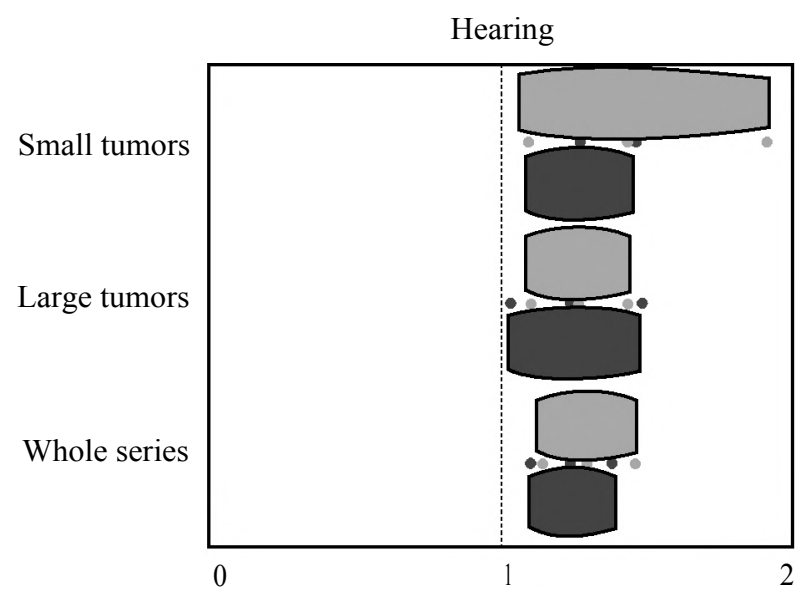

Facial nerve

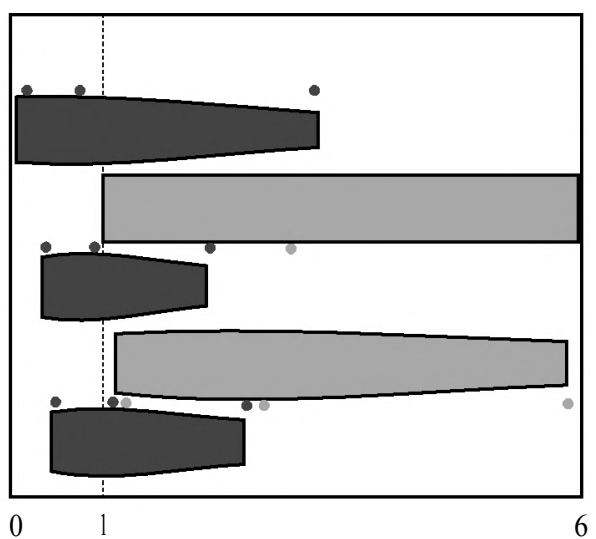

Tinnitus

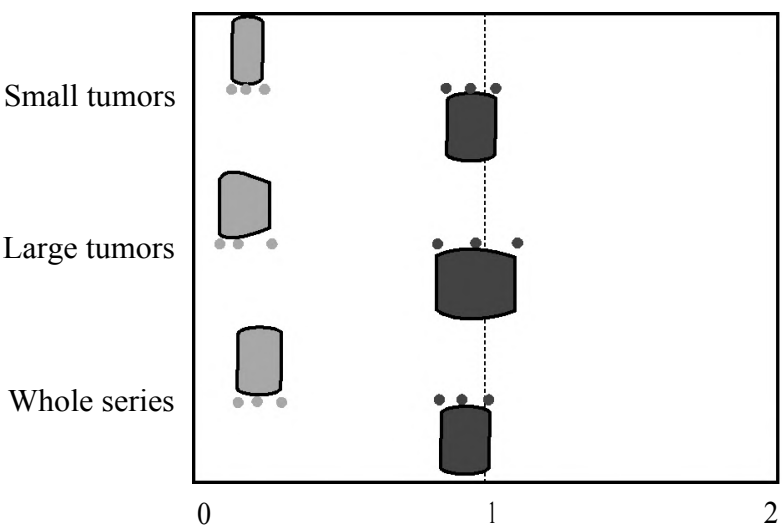

Facial hypoesthesia

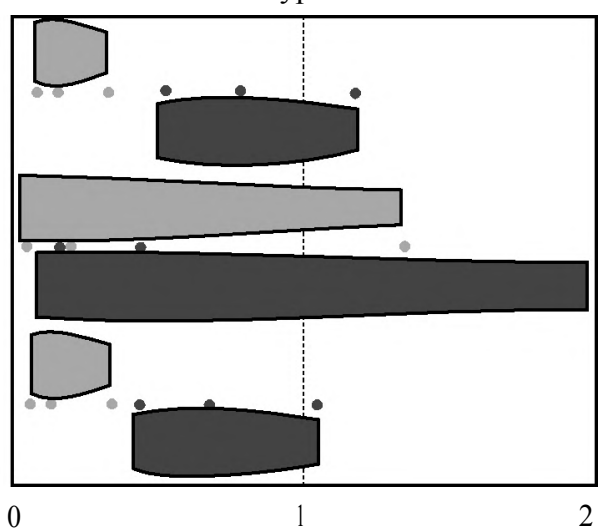

Dizziness
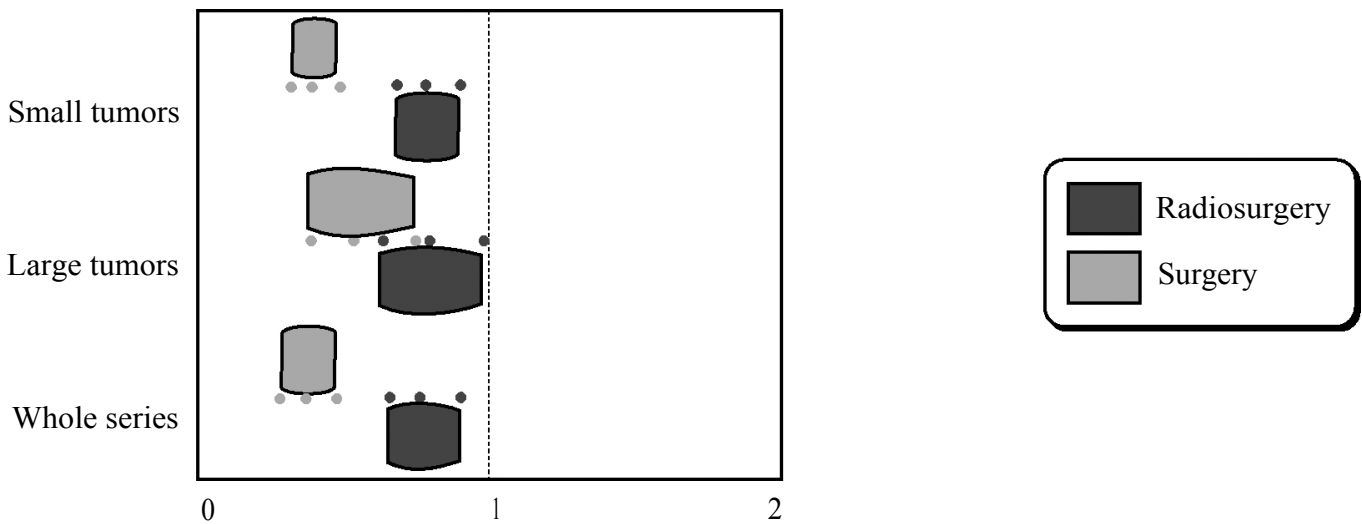

Fig. (2): Relative risk for developing a new neurologic deficit in either group (radiosurgery in blue and surgery in pink). Values below 1 represent a protective effect, whereas values above 1 indicate the relative risk of developing a deficit. For hearing (risk of developing non-serviceable hearing, or Gardner-Robertson value $>2$ ), facial nerve function (risk of developing a visible facial nerve palsy, i.e., House-Brackmann $>2$ ). 

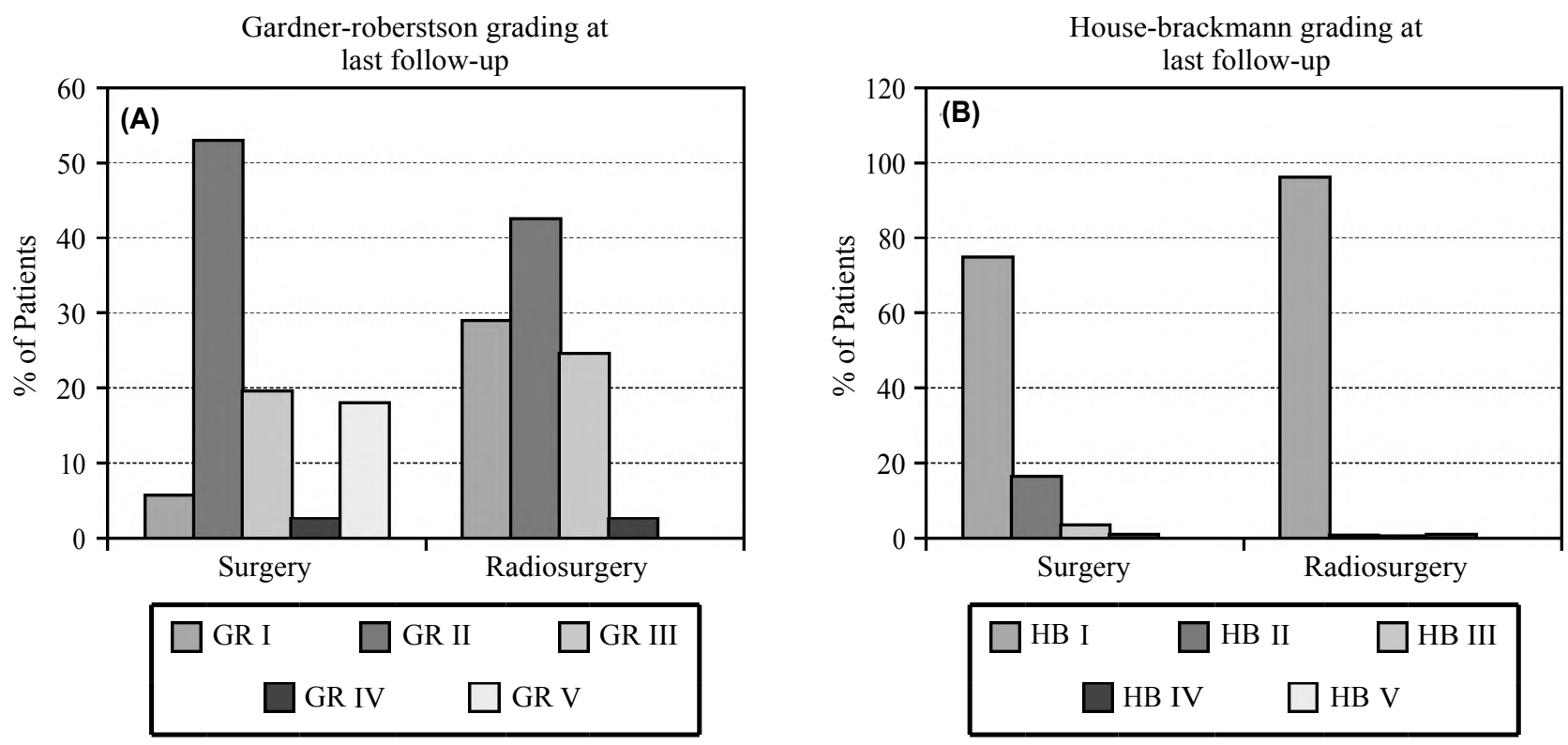

Fig. (3): (A) Proportion of patients with serviceable hearing after treatment (G\&R grades I and II). For this analysis, only cases with serviceable hearing pre-treatment were included. (B) Proportion of patients with non-apparent facial nerve deficit (HB grades I and II) after treatment (only included patients without apparent facial palsy prior to treatment).

Hearing function according to tumor size
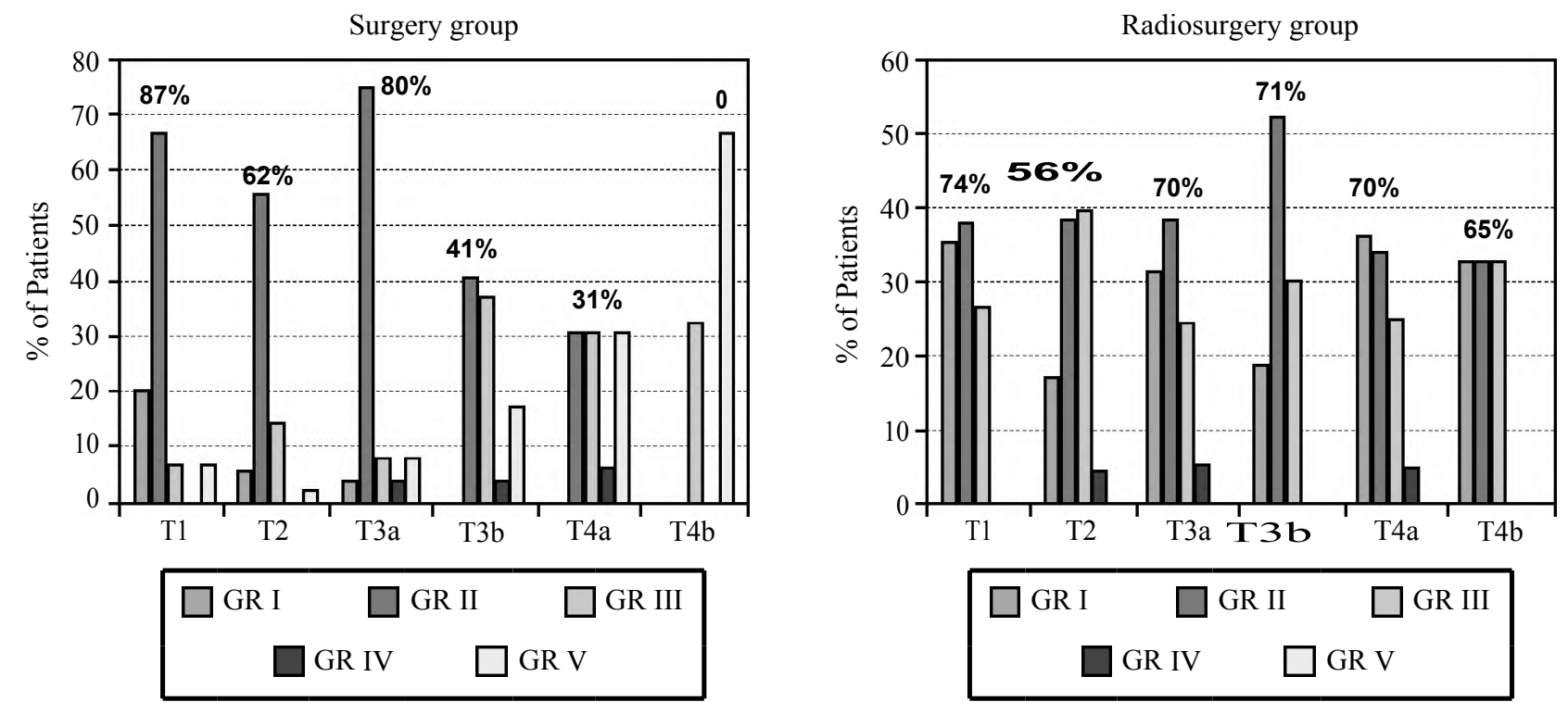

Fig. (4): Hearing function after treatment in relation to tumor size according to the Hannover classification. The percentages at the top of the bars indicate the proportion of patients with serviceable hearing (GR grades I and II) at the latest follow-up. 
Facial function according to tumor size
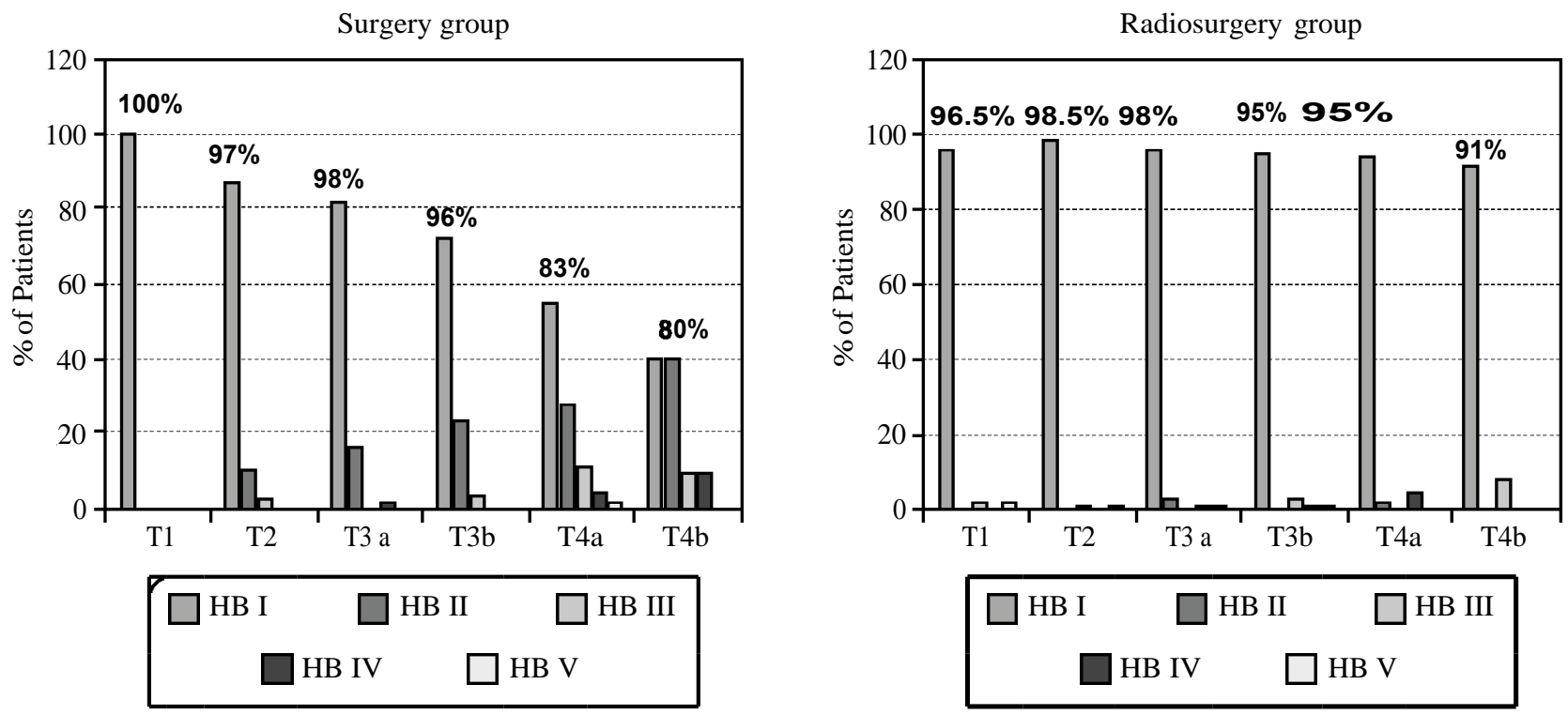

Fig. (5): Facial nerve function after treatment in relation to tumor size according to the Hannover classification. The percentages at the top of the bars indicate the proportion of patients with non-visible facial nerve deficit (HB grades I \& II) at the latest follow-up.

\section{Discussion}

Although the outcome after radiosurgery [6-8] or surgery [9-11] for VSs has been described in various reports, comparative studies offering level 1 or 2 evidence are still lacking [12]. Large VSs are usually approached surgically to relieve the compression on the brainstem [13]. The choice of management for small and medium-sized tumors, on the other hand, is highly controversial $[\mathbf{1 4 , 1 5}]$.

\section{Evolution of surgery results:}

Despite recent advances in neurosurgery, neurophysiological monitoring, neuroanesthesia, and intensive care, a recent review of surgical series from the last 15 years revealed that there are still considerable morbidity and mortality rates. Specifically, facial nerve palsy was observed in $8 \%$ to $68.6 \%$ of surgery cases (mean: $34.0 \%$ ), while hearing preservation rates ranged from $24.6 \%$ to $54.1 \%$ [5,16-18]. In this study, we compared a single surgeon's experience (MT) with results from a reference center for gamma knife radiosurgery.

\section{Radiosurgery tumor control results:}

Two large series of patients submitted to gamma knife radiosurgery reported better facial nerve function and hearing preservation relative to surgery $[7,19]$. Although many reports on functional outcome after radiosurgery can be found in the literature, only one study mathematically assessed tumor shrinkage after treatment [19]. In this recent study, the authors compared the follow-up of 113 patients who had undergone $\mathrm{g}$ knife radiosurgery with that of 124 patients managed conservatively (follow-up 55 months). Similarly to our own study, these authors assessed tumor size at follow-up normalized to the pre-treatment value. They reported a relative size of $0.85 \pm 0.61$ (indicating a reduction of $15 \%$ at the last follow-up), as compared with $1.83 \pm 2.44$ in the conservative arm (83\% increment). Although they did not apply a linear model, the authors calculated the doubling time as 3.3 years in the conservative arm, versus 14.5 years in the radiosurgery group.

\section{Comparative studies:}

To date, very few comparative studies between radiosurgery and microsurgery have been published [20-25]. In 1995, Pollock [20] reported on the Pittsburgh experience with 47 patients treated by radiosurgery and 40 managed surgically, all harboring small VSs $(<3 \mathrm{~cm})$. These patients were treated between 1990 and 1991 and were followed-up for 36 months (25-48). In that study, radiosurgery yielded better results than surgery for hearing preservation and facial nerve function $(p<0.05$ for both), with lower morbidity $(p<0.01)$. Interestingly, patient satisfaction and overall functional outcome did not differ between groups. Similar observations were reported by a Dutch-Swedish study involving 145 patients [21]. According to those authors, overall health self-rating (assessed via questionnaires sent to the patients) was better after radiosurgery. 
Similarly, a group from Marseille [23] reported their experience in which they compared the first 97 patients submitted to gamma knife radiosurgery to a historical cohort of 110 patients from the same institution who had undergone micro surgical treatment prior to 1992. Surprisingly, they reported no new facial deficits among radiosurgery patients and new deficits in $47 \%$ of surgery patients. Moreover, they reported hearing loss in $62.5 \%$ of radiosurgery patients and 30\% of surgery patients (both differences were statistically significant). Finally, radiosurgery proved to be superior for facial hypoesthesia, ocular problems, dysphagia, and return to work. Regarding tinnitus and vertigo, no differences between groups were observed.

The findings are very different from those reported in the current study. Overall, we confirmed the superiority of radiosurgery over surgery regarding facial nerve function and hearing. However, our data show that the greatest difference between the two groups is the higher percentage of surgery cases who were initially HB I and evolved to HB II. However, these deficits were usually mild and did not significantly impair patients' quality of life. Noteworthy, facial nerve function tends to remain stable after irradiation. One might assume that these results are influenced by tumor size. In fact, separate analyses of small and large tumor groups confirmed a higher relative risk of facial nerve damage associated with surgery in the large tumor population. In the small tumor group, however, the absence of pre-operative facial palsy makes it impossible to calculate relative risk. In the radiosurgery group, on the other hand, all risk intervals (for both the small and large tumor groups) included the value " $1 "$, thus confirming a trend towards stability.

In terms of hearing preservation, radiosurgery seems to be preferable to surgery, as assessed at both the short and long follow-ups. The lack of recovery in the surgical series indicates that the cochlear nerve may be especially sensitive to surgical manipulation and thus unable to recover. Notwithstanding, in the case of small tumors, the advantage of radiosurgery over surgery seems to fade away, as both treatments rendered similar functional results. Interestingly, the relative risks were also similar between the small and large tumor groups.

One difference between groups was a wider risk interval for hearing deterioration in small tumors submitted to surgery. This might reflect the fact that even small tumors located deep in the meatus may be at risk for hearing deterioration.
The apparent discrepancy between relative risk calculations and outcome comparisons between groups may be explained by the fact that the study arms differed significantly in terms of tumor size; in other words, some extent of the "protective effect" of radiosurgery may be due to the fact that patients treated with radiosurgery usually harbor smaller tumors. Another more plausible explanation could be that the analyses conducted are not the same: While the outcome comparison reflects changes in functional status, the relative risk depicts the risk of developing either an apparent facial palsy or non-serviceable hearing. These two outcome measures better reflect the risk of developing clinically significant functional changes. In fact, the mean changes in the grading scales observed after treatments were very discrete and only statistically relevant because of the large number of patients enrolled in this study.

In contrast to what has been reported by others [23], we observed a protective effect of surgery for vertigo, facial hypoesthesia, and tinnitus. This proved to be true for both small and large tumors. In the case of trigeminal nerve function, the recovery observed may be a direct consequence of the surgical decompression. On the other hand, the slow decompression rate that follows radiosurgery may not be fast enough to permit regeneration of the trigeminal nerve. We believe that the clinical improvement observed for tinnitus and vertigo might be due to complete damage of the vestibular and cochlear nerves, which at times is not avoidable during surgery. Damage to the cochlear nerve abolishes tinnitus abruptly, while damage to the vestibular nerve causes a transient and acute amelioration of vertigo, which is rapidly compensated in the post-operative period.

Karpinos et al., [22] report on their experience in Houston with 96 patients treated from 1993 to 2000. Follow-up was 2 years for the surgery arm and 4 years for the radiosurgery group. The study populations differed with respect to size and age (radiosurgery was more often used for smaller tumors and older patients). Noteworthy, a large proportion of patients in this series were treated through a trans-labyrinthine approach, which explains the low rate of hearing preservation after surgery $(14.4 \%$, compared to $57.5 \%$ after radiosurgery). A functional advantage of radiosurgery over microsurgery was also reported for facial hypoesthesia and facial palsy. Moreover, no differences related to dizziness and tinnitus were observed. The findings from this series also differed significantly from our own, which might be partly explained by the small number of patients enrolled, 
the relatively short follow-up period, and the variety of surgical approaches performed in their study. In terms of tumor control after radiosurgery, the authors reported an increase in tumor size in $9 \%$ of patients, stability in $54.5 \%$ and reduction in $36.4 \%$. In our radiosurgery arm, by contrast, we observed tumor growth in $35 \%$ of patients at followup. This discrepancy probably reflects the longer observation time in our series, together with a more sensitive method of assessing tumor volume.

In another recent retrospective study from Norway, Myrseth et al., [24] reported on 189 patients: 86 submitted to microsurgery and 103 to radiosurgery. Tumor reduction (defined as at least $30 \%$ shrinkage) was observed in $49 \%$ of patients after radiosurgery, stability ( $70 \%$ to $140 \%$ of the initial size) in $40.2 \%$, and tumor growth in $10.8 \%$, after a mean follow-up of 5.9 years. Treatment failure (i.e., growth of more than $40 \%$ or the need for additional treatment) was similar in both groups. Although the observation time was similar to that in our study (up to 12 years), tumor control rate was not objectively assessed.

It should be noted that most of the comparative studies reviewed refer to old series, and the more recent studies mostly included small patient groups or had short observation times. Additionally, several of these studies used weak criteria for defining tumor control, which precludes any robust conclusions about the efficacy of each treatment modality. In this sense, our study represents an important contribution to the field, by providing recent evidence about the efficacy of radiosurgery and microsurgery for the treatment of vestibular schwannomas, considering the modern advances in both the surgical and radiosurgical techniques. We calculated the relative risks associated with each treatment modality and objectively evaluated the trend for a reduction in tumor volume after radiosurgery. According to our model, a reduction of $50 \%$ can be reached, on average, after 12 years.

\section{Conclusion}

In conclusion, radiosurgery caused less morbidity and was more effective for the preservation of facial nerve function, both for small and large tumors. The facial nerve deterioration seen after surgery was, however, not clinically relevant. In addition, radiosurgery resulted in relatively betterpreserved hearing, but only in the case of large tumors. On the other hand, surgery resulted in relatively better outcome in terms of tinnitus, vertigo, and facial hypoesthesia. In terms of tumor control, radiosurgery was effective in $87 \%$ of cases who had either stable or reduced tumors (however, in $35 \%$ of cases, tumors had grown by the time of the long-term follow-up). We also observed an overall trend for tumor reduction after radiosurgery. We should note that the present study is a retrospective analysis conducted in two different centers. Despite the high number of patients and long follow-up, the analysis is limited by the fact that the study arms were not randomized, and thus it carries an unavoidable selection bias, since patients with larger tumors are more often referred to surgical treatment, whereas small tumors are more often irradiated. To overcome this limitation, we stratified the study groups by tumor size (small vs. large tumors).

In conclusion, our results suggest that looking for the "best treatment option" should involve combining everything we know about each approach with each patient's particular expectations, wishes, life expectancy, co-morbidities, and professional activity.

\section{References}

1- FLINT D., FAGAN P. and PANARESE A.: Conservative management of sporadic unilateral acoustic neuromas. The Journal of Laryngology and Otology. Jun., 119 (6): 424-428, 2005.

2- ARTHURS B.J., FAIRBANKS R.K., DEMAKAS J.J., et al.: A review of treatment modalities for vestibular schwannoma. Neurosurgical Review. Jul., 34 (3): 265-277; discussion 277-269, 2011.

3- CHARABI S., TOS M., THOMSEN J., CHARABI B. and MANTONI M.: Vestibular schwannoma growth--longterm results. Acta oto-laryngologica. Supplementum. 543: 7-10. 2000.

4- KOOS W.T., DAY J.D., MATULA C. and LEVY D.I.: Neurotopographic considerations in the microsurgical treatment of small acoustic neurinomas. Journal of Neurosurgery. Mar., 88 (3): 506-512, 1998.

5- SAMII M. and MATTHIES C.: Management of 1000 vestibular schwannomas (acoustic neuromas): Surgical management and results with an emphasis on complications and how to avoid them. Neurosurgery. Jan., 40 (1): 11-21, discussion 21-13, 1997.

6- FLICKINGER J.C., KONDZIOLKA D., NIRANJAN A., MAITZ A., VOYNOV G. and LUNSFORD L.D.: Acoustic neuroma radiosurgery with marginal tumor doses of 12 to 13 Gy. International Journal of Radiation Oncology, Biology, Physics. Sep., 1, 60 (1): 225-230, 2004.

7- HASEGAWA T., FUJITANI S., KATSUMATA S., KIDA Y., YOSHIMOTO M. and KOIKE J.: Stereotactic radiosurgery for vestibular schwannomas: Analysis of 317 patients followed more than 5 years. Neurosurgery Aug., 57 (2): 257-265; discussion 257-265, 2005.

8- IWAI Y., YAMANAKA K., SHIOTANI M. and UYAMA T.: Radiosurgery for acoustic neuromas: Results of lowdose treatment. Neurosurgery Aug., 53 (2): 282-287, discussion 287-288, 2003. 
9- BETCHEN S.A., WALSH J. and POST K.D.: Long-term hearing preservation after surgery for vestibular schwannoma. Journal of Neurosurgery. Jan., 102 (1): 6-9, 2005.

10- BRACKMANN D.E., OWENS R.M., FRIEDMAN R.A., et al.: Prognostic factors for hearing preservation in vestibular schwannoma surgery. The American Journal of Otology. May., 21 (3): 417-424, 2000.

11- SAMII M. and MATTHIES C.: Management of 1000 vestibular schwannomas (acoustic neuromas): Hearing function in 1000 tumor resections. Neurosurgery. Feb., 40 (2): 248-260, discussion 260-242, 1997.

12-NIKOLOPOULOS T.P. and O'DONOGHUE G.M.: Acoustic neuroma management: an evidence-based medicine approach. Otology \& neurotology: Official publication of the American Otological Society, American Neurotology Society [and] European Academy of Otology and Neurotology. Jul., 23 (4): 534-541, 2002.

13- NONAKA Y., FUKUSHIMA T., WATANABE K., et al. Less invasive transjugular approach with Fallopian bridge technique for facial nerve protection and hearing preservation in surgery of glomus jugulare tumors. Neurosurgical review. Oct., 36 (4): 579-586, discussion 586, 2013.

14- KAYLIE D.M., HORGAN M.J., DELASHAW J.B. and MCMENOMEY S.O.: A meta-analysis comparing outcomes of microsurgery and gamma knife radiosurgery. The Laryngoscope. Nov., 110 (11): 1850-1856, 2000.

15- POLLOCK B.E., LUNSFORD L.D. and NOREN G.: Vestibular schwannoma management in the next century: a radiosurgical perspective. Neurosurgery. Sep., 43 (3): 475-481, discussion 481-473, 1998.

16- CHARPIOT A., TRINGALI S., ZAOUCHE S., FERBERVIART C. and DUBREUIL C.: Perioperative complications after translabyrinthine removal of large or giant vestibular schwannoma: Outcomes for 123 patients. Acta oto-laryngologica. Nov., 130 (11): 1249-1255, 2010.

17- SAMII M., GERGANOV V. and SAMII A.: Improved preservation of hearing and facial nerve function in vestibular schwannoma surgery via the retrosigmoid approach in a series of 200 patients. Journal of Neurosurgery. Oct., 105 (4): 527-535, 2006.

18- SUGHRUE M.E., YANG I., ARANDA D., et al.: Beyond audiofacial morbidity after vestibular schwannoma surgery. Journal of Neurosurgery. Feb., 114 (2): 367-374, 2011.

19- BREIVIK C.N., NILSEN R.M., MYRSETH E., et al.: Conservative management or gamma knife radiosurgery for vestibular schwannoma: Tumor growth, symptoms, and quality of life. Neurosurgery. Jul., 73 (1): 48-56, discussion 56-47, 2013.

20- POLLOCK BE, LUNSFORD LD, KONDZIOLKA D, et al.: Outcome analysis of acoustic neuroma management: A comparison of microsurgery and stereotactic radiosurgery. Neurosurgery. Jan., 36 (1): 215-224, discussion 224 $219,1995$.

21- VAN ROIJEN L., NIJS H.G., AVEZAAT C.J., et al.: Costs and effects of microsurgery versus radiosurgery in treating acoustic neuroma. Acta. neurochirurgica, 139 (10): 942-948, 1997.

22- KARPINOS M., TEH B.S., ZECK O., et al.: Treatment of acoustic neuroma: stereotactic radiosurgery vs. microsurgery. International Journal of Radiation Oncology, Biology, Physics. Dec., 1, 54 (5): 1410-1421, 2002.

23- REGIS J., PELLET W., DELSANTI C., et al.: Functional outcome after gamma knife surgery or microsurgery for vestibular schwannomas. Journal of Neurosurgery. Nov., 97 (5): 1091-1100, 2002.

24- MYRSETH E., MOLLER P., PEDERSEN P.H., VASSBOTN F.S., WENTZEL-LARSEN T. and LUNDJOHANSEN M.: Vestibular schwannomas: Clinical results and quality of life after microsurgery or gamma knife radiosurgery. Neurosurgery. May, 56 (5): 927-935, discussion 927-935, 2005.

25- POLLOCK B.E., DRISCOLL C.L., FOOTE R.L., et al.: Patient outcomes after vestibular schwannoma management: A prospective comparison of microsurgical resection and stereotactic radiosurgery. Neurosurgery. Jul., 59 (1): 77-85, discussion 77-85, 2006. 


\section{علاج أورام العصب المخى الثامن

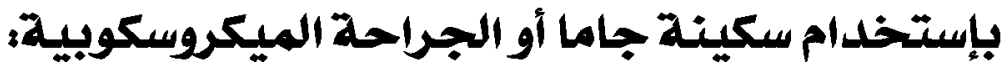

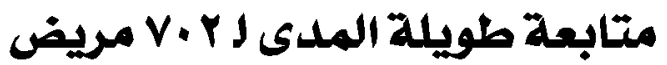

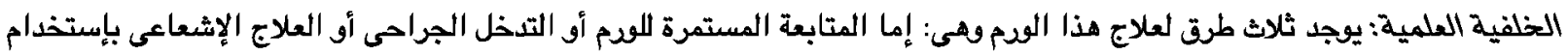
الجراحة الإشعاعية المجسمة. لا يوجد إلى الآن إثبات لأفضلية إحدى هذى هذه الطفي الطرق.

الهدف من الدراسة: مقارنة النتائج طويلة المدى لعلاج هذا الوقم بإستخدام الجراحة الميكرسكوبية وسكينة جاما.

المرضى وطرق العلاج: تم علاج هVYمريض بالجراحة الميكروسكوبية، VYع مريض بإستخدام سكينة جاما أعتمدت المقارنة بين هاتين

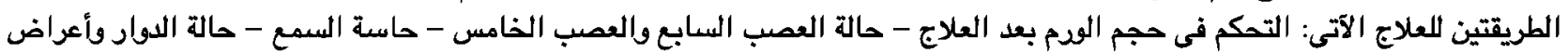
طنين الآذن.

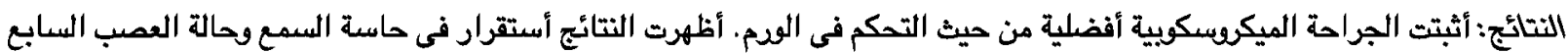

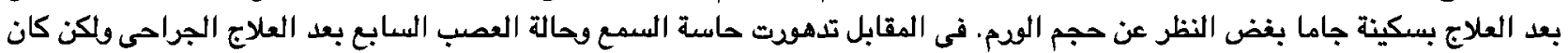

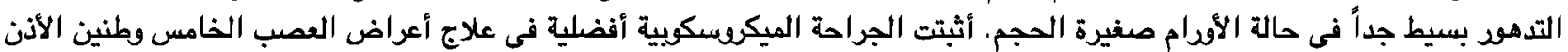
وكنلك في علاج الدوار.

الخلاصدة: تمثل هذه النتائج إضافة مهمة فى هذا المجال وتقدم توصيات قائمة على الدليل أفضلية إحدى طرق العلاج على الأخرى بناءاً على توقعات المرضى وأنشطتهم الوظيفية . 